\title{
LOWER BOUNDS FOR BLOW-UP TIME IN SOME NON-LINEAR PARABOLIC PROBLEMS UNDER NEUMANN BOUNDARY CONDITIONS
}

\author{
CRISTIAN ENACHE \\ Department of Mathematics and Informatics, Ovidius University, Constanta, 900597, Romania \\ e-mail: cenache@univ-ovidius.ro
}

(Received 13 May 2010; accepted 21 September 2010; first published online 10 March 2011)

\begin{abstract}
This paper deals with some non-linear initial-boundary value problems under homogeneous Neumann boundary conditions, in which the solutions may blow up in finite time. Using a first-order differential inequality technique, lower bounds for blow-up time are determined.
\end{abstract}

2010 Mathematics Subject Classification. 35K20, 35B45, 35B30.

1. Introduction. The study of the blow-up phenomena in parabolic problems has received a great deal of attention in the last decades (we refer the reader especially to the books of Straughan [12] and Quittner-Souplet [11], the survey papers of Levine [4] and Galaktionov [2] and the references therein). Therefore, nowadays a variety of methods are known and used in the study of various questions regarding the blow-up phenomena in parabolic problems. But, most of the methods used to show that solutions blow-up provide only an upper bound for the blow-up time, while in applications, due to the explosive nature of the solutions, it is more important to determine the lower bounds on the blow-up time. We note, however, that during the last four years, beginning with the paper of Payne and Schaefer [6], such lower bounds on blow-up time have been obtained in various parabolic problems, by mean of a first-order differential inequality technique (see, for instance, [5]-[9] and some references therein).

In this paper, we will consider the following type of non-linear parabolic problems in divergence form:

$$
\begin{cases}\left(\rho\left(\mathbf{x}, u,|\nabla u|^{2}\right) u_{, i}\right)_{, i}-u_{, t}=-f(u) & \text { in } \Omega \times\left(0, t^{*}\right), \\ \frac{\partial u}{\partial n}=0 & \text { on } \partial \Omega \times\left(0, t^{*}\right), \\ u(\mathbf{x}, 0)=g(\mathbf{x}) \geq 0 & \text { in } \Omega,\end{cases}
$$

where $u_{, t}$ denotes the partial derivative of $u(\mathbf{x}, t)$ with respect to $t$, the symbol ${ }_{, i}$ denotes the partial differentiation with respect to $x_{i}, i=1,2,3, \partial u / \partial n$ is the outward normal derivative of $u(\mathbf{x}, t)$ on the boundary $\partial \Omega$ and the summation is understood on repeated indices. Moreover, the domain $\Omega \subset \mathbb{R}^{3}$ is assumed to be bounded, starshaped, convex in two orthogonal directions and with smooth boundary $\partial \Omega$, while $\rho$ is a positive $C^{1}$ function that satisfies the ellipticity condition throughout $\Omega$, i.e.

$$
\rho(\mathbf{x}, u, s)+2 s \frac{\partial}{\partial s} \rho(\mathbf{x}, u, s)>0, \quad s>0, \mathbf{x} \in \Omega .
$$


We also ask that $\rho$ and $f$ satisfy the conditions

$$
0<f(s) \leq a_{1}+a_{2} s^{p}, \quad \rho(\mathbf{x}, u, s) \geq b_{1}, \quad s>0, \mathbf{x} \in \Omega,
$$

where $p>1$ and $a_{1} \in \mathbb{R}_{+}, a_{2}, b_{1} \in \mathbb{R}_{+}^{*}$. In addition, $g$ is assumed to satisfy the compatibility condition $\partial g / \partial n=0$ on $\partial \Omega$. Under these assumptions on the data, it follows from the parabolic maximum principles (see Protter-Weinberger [10]) that the solution of the problem (1.1) is non-negative. Moreover, it is well-known that the solution may not exist for all time, and the only way that it can fail to exist is by becoming unbounded at some finite time $t^{*}$ (see, for instance, the works of Ball [1] and Kielhöfer [3] in the case $\rho \equiv 1)$. This phenomena depends on the form of $f(u)$ and $\rho\left(\mathbf{x}, u,|\nabla u|^{2}\right)$, the initial data $g(\mathbf{x})$ or the geometry of the given domain $\Omega$.

In what follows, we shall assume that a non-negative classical solution of the problem (1.1)-(1.3) exists and become unbounded at time $t=t^{*}$. Our aim is to determine an explicit lower bound for the the blow-up time $t^{*}$ in some appropriate measure. We notice that lower bounds for blow-up time in non-linear parabolic problems with particular divergence form, but under Dirichlet boundary conditions and different assumptions on the data, have been recently obtained by PaynePhilippin-Schaefer in [5]. A key ingredient in their proof was the Sobolev inequality, which is no longer applicable in our case, since we deal with homogeneous Neumann boundary conditions. However, for a class of semi-linear heat equations under homogeneous Neumann boundary conditions, Payne and Schaefer succeeded [6] to overpass this difficulty by the determination of an appropriate Sobolev-type inequality for $C^{1}$-functions. In order to handle the more general problem (1.1)-(1.3), our approach is inspired by their technique, the main ingredient of our argument being again the determination of an appropriate Sobolev-type inequality for $\mathrm{C}^{1}$-functions on $\Omega$.

2. Lower bound on blow-up time. Let us introduce the auxiliary function

$$
\Phi(t):=\int_{\Omega} u^{2 n} d x
$$

for some constant $n>1$ to be chosen. We compute

$$
\begin{aligned}
\Phi^{\prime}(t) & =2 n \int_{\Omega} u^{2 n-1}\left[\left(\rho\left(\mathbf{x}, u,|\nabla u|^{2}\right) u_{, i}\right)_{, i}+f(u)\right] d x \\
& =-2 n(2 n-1) \int_{\Omega} u^{2 n-2} \rho\left(\mathbf{x}, u,|\nabla u|^{2}\right)|\nabla u|^{2} d x+2 n \int_{\Omega} u^{2 n-1} f(u) d x \\
& \leq-2 n(2 n-1) b_{1} \int_{\Omega} u^{2 n-2}|\nabla u|^{2} d x+2 n \int_{\Omega} u^{2 n-1}\left(a_{1}+a_{2} u^{p}\right) d x,
\end{aligned}
$$

where we have used successively the differential equation (1.1), the divergence theorem, the boundary condition (1.1) and the assumption (1.3). Next, we notice that

$$
\left|\nabla u^{n}\right|^{2}=n^{2} u^{2(n-1)}|\nabla u|^{2},
$$

and we use Holder's inequality to obtain

$$
\Phi^{\prime}(t) \leq-\frac{2 n(2 n-1)}{n^{2}} b_{1} \int_{\Omega}\left|\nabla u^{n}\right|^{2} d x+2 n a_{1}|\Omega|^{\frac{1}{2 n}} \Phi(t)^{\frac{2 n-1}{2 n}}+2 n a_{2} \int_{\Omega} u^{2 n+p-1} d x .
$$


Now, our aim is to transform the right side of (2.4) in terms of $\Phi(t)$ and obtain a first-order differential inequality for $\Phi$. To accomplish this, we begin by using Holder's inequality to write:

$$
\int_{\Omega} u^{2 n+p-1} d x \leq\left(\int_{\Omega} u^{4 n} d x\right)^{\frac{1}{3}}\left(\int_{\Omega} u^{\frac{2 n+3 p-3}{2}} d x\right)^{\frac{2}{3}} .
$$

To bound the integral of $u^{(2 n+3 p-3) / 2}$, we use again Holder's inequality and obtain

$$
\int_{\Omega} u^{\frac{2 n+3 p-3}{2}} d x \leq|\Omega|^{1-\mu}|\Phi(t)|^{\mu}, \quad \text { with } \quad \mu:=\frac{2 n+3 p-3}{4 n},
$$

where $|\Omega|$ denotes the volume of $\Omega$ and, in order to ensure that $\mu<1$ in (2.6), the constant $n$ must be chosen to satisfy $n>3(p-1) / 2$.

Next, to bound the integral of $u^{4 n}$ in (2.5), we seek to determine an appropriate Sobolev-type inequality. For this aim, we denote by $x_{i m}$ and $x_{i M}$ the minimum and the maximum values, respectively, of the coordinates $x_{i}, i=1,2,3$, relative to $\Omega$ and by $v_{i}, i=1,2,3$, the components of the unit outer normal to $\partial \Omega$. We also denote by $D_{z}$ the intersection of $\Omega$ with the plane $x_{3}=z$ and, for clarity, we let $w:=u^{n}$. Then, using Schwarz's inequality, we can write

$$
\int_{\Omega} w^{4} d x=\int_{x_{3 m}}^{x_{3 M}}\left(\int_{D_{z}} w^{4} d A\right) d \xi \leq \int_{x_{3 m}}^{x_{3 M}}\left[\int_{D_{z}} w^{2} d A \int_{D_{z}} w^{6} d A\right]^{\frac{1}{2}} d \xi .
$$

Now, let $\mathbf{P}=\left(\bar{x}_{1}, \bar{x}_{2}, z\right)$ be an arbitrary point in $D_{z}$ and $\mathbf{P}_{1}:=\left(\xi_{1}, \bar{x}_{2}, z\right)$ and $\mathbf{P}_{2}:=$ $\left(\xi_{2}, \bar{x}_{2}, z\right)$ denotes the points on the boundary $\partial D_{z}$ where the line $x_{2}=\bar{x}_{2}$ in $D_{z}$ intersects the boundary $\partial D_{z}$. Similarly, let $\mathbf{Q}_{1}:=\left(\bar{x}_{1}, \eta_{1}, z\right)$ and $\mathbf{Q}_{2}:=\left(\bar{x}_{1}, \eta_{2}, z\right)$ be the points on the boundary $\partial D_{z}$, where the line $x_{2}=\bar{x}_{2}$ in $D_{z}$ intersects $\partial D_{z}$. We then have

$$
\begin{aligned}
& w^{3}(\mathbf{P})=w^{3}\left(\mathbf{P}_{1}\right)+3 \int_{\mathbf{P}_{1}}^{\mathbf{P}} w^{2} w_{, 1} d x_{1}, \\
& w^{3}(\mathbf{P})=w^{3}\left(\mathbf{P}_{2}\right)-3 \int_{\mathbf{P}_{2}}^{\mathbf{P}} w^{2} w_{, 1} d x_{1},
\end{aligned}
$$

from which we obtain

$$
w^{3}(\mathbf{P}) \leq \frac{1}{2}\left[w^{3}\left(\mathbf{P}_{1}\right)+w^{3}\left(\mathbf{P}_{2}\right)\right]+\frac{3}{2} \int_{\mathbf{P}_{1}}^{\mathbf{P}_{2}} w^{2}\left|w_{, 1}\right| d x_{1} .
$$

In a similar way, one may show that

$$
w^{3}(\mathbf{P}) \leq \frac{1}{2}\left[w^{3}\left(\mathbf{Q}_{1}\right)+w^{3}\left(\mathbf{Q}_{2}\right)\right]+\frac{3}{2} \int_{\mathbf{Q}_{1}}^{\mathbf{Q}_{2}} w^{2}\left|w_{, 2}\right| d x_{2} .
$$

Therefore, multiplying (2.9) and (2.10) and integrating over $D_{z}$, we get

$$
\begin{aligned}
\int_{D_{z}} w^{6} d A & \leq \frac{1}{4}\left\{\int_{x_{2 m}}^{x_{2 M}}\left[w^{3}\left(\mathbf{P}_{1}\right)+w^{3}\left(\mathbf{P}_{2}\right)\right] d x_{2}+3 \int_{D_{z}} w^{2}\left|w_{,_{1}}\right| d A\right\} . \\
& \cdot\left\{\int_{x_{1 m}}^{x_{1 M}}\left[w^{3}\left(\mathbf{Q}_{1}\right)+w^{3}\left(\mathbf{Q}_{2}\right)\right] d x_{1}+3 \int_{D_{z}} w^{2}\left|w_{, 2}\right| d A\right\} .
\end{aligned}
$$


Next, making use of the fact that

$$
\begin{aligned}
& \int_{x_{2 m}}^{x_{2 M}}\left[w^{3}\left(\mathbf{P}_{1}\right)+w^{3}\left(\mathbf{P}_{2}\right)\right] d x_{2} \leq \int_{\partial D_{z}} w^{3}\left|v_{1}\right| d s, \\
& \int_{x_{1 m}}^{x_{1 M}}\left[w^{3}\left(\mathbf{Q}_{1}\right)+w^{3}\left(\mathbf{Q}_{2}\right)\right] d x_{1} \leq \int_{\partial D_{z}} w^{3}\left|v_{2}\right| d s,
\end{aligned}
$$

together with the facts that $\left|v_{k}\right|<1,\left|w_{, k}\right|<|\nabla w|, k=1,2$, and Schwarz's inequality, it follows from (2.11) that

$$
\int_{D_{z}} w^{6} d A \leq \frac{1}{4}\left\{\int_{\partial D_{z}} w^{3} d s+3\left[\int_{D_{z}} w^{4} d A \int_{D_{z}}|\nabla w|^{2} d A\right]^{\frac{1}{2}}\right\}^{2}
$$

Therefore, making use of Schwarz's inequality and (2.13), we deduce that

$$
\int_{D_{z}} w^{4} d A \leq \frac{1}{2}\left[\max _{z} \int_{D_{z}} w^{2} d A\right]^{\frac{1}{2}}\left\{\int_{\partial D_{z}} w^{3} d s+3\left[\int_{D_{z}} w^{4} d A \int_{D_{z}}|\nabla w|^{2} d A\right]^{\frac{1}{2}}\right\}
$$

Integrating now (2.14) over $z$ we get

$$
\int_{\Omega} w^{4} d x \leq \frac{1}{2}\left[\max _{z} \int_{D_{z}} w^{2} d A\right]^{\frac{1}{2}}\left\{\int_{\partial \Omega} w^{3} d s+3\left[\int_{\Omega} w^{4} d x \int_{\Omega}|\nabla w|^{2} d x\right]^{\frac{1}{2}}\right\},
$$

where we have used Schwarz's inequality to obtain the last term.

We now seek to bound $\int_{\partial \Omega} w^{3} d s$ and $\max _{z} \int_{D_{z}} w^{2} d A$. For this aim, we denote by

$$
p_{0}:=\min _{\partial \Omega}(\mathbf{x} \cdot \mathbf{n}), \quad d^{2}:=\max _{\Omega}|\mathbf{x}|
$$

and make use of the divergence theorem to write

$$
p_{0} \int_{\partial \Omega} w^{3} d s \leq \int_{\partial \Omega} x_{i} n_{i} w^{3} d s=3 \int_{\Omega} w^{3} d x+3 \int_{\Omega} x_{i} w^{2} w_{, i} d x
$$

It then follows that

$$
\int_{\partial \Omega} w^{3} d s \leq \frac{3}{p_{0}} \int_{\Omega} w^{3} d x+\frac{3 d}{p_{0}}\left[\int_{\Omega} w^{4} d x \int_{\Omega}|\nabla w|^{2} d x\right]^{\frac{1}{2}}
$$

where we have used Schwarz's inequality to get the last term. Replacing (2.18) in (2.15), we obtain 


$$
\begin{aligned}
\int_{\Omega} w^{4} d x \leq & \frac{3}{2}\left[\max _{z} \int_{D_{z}} w^{2} d A\right]^{\frac{1}{2}}\left\{\frac{1}{p_{0}} \int_{\Omega} w^{3} d x+\left(1+\frac{d}{p_{0}}\right)\left[\int_{\Omega} w^{4} d x \int_{\Omega}|\nabla w|^{2} d x\right]^{\frac{1}{2}}\right\} \\
\leq & \frac{3}{2}\left[\max _{z} \int_{D_{z}} w^{2} d A\right]^{\frac{1}{2}}\left(\int_{\Omega} w^{4} d x\right)^{\frac{1}{2}} \\
& \times\left\{\frac{1}{p_{0}}\left(\int_{\Omega} w^{2} d x\right)^{\frac{1}{2}}+\left(1+\frac{d}{p_{0}}\right)\left(\int_{\Omega}|\nabla w|^{2} d x\right)^{\frac{1}{2}}\right\},
\end{aligned}
$$

where we have used again Schwarz's inequality to get the last expression.

Next, in order to bound $\max _{z} \int_{D_{z}} w^{2} d A$ in (2.19), we let $\Omega^{+}$be the portion of $\Omega$ above $D_{z}$, with $\partial \Omega^{+}$the portion of $\partial \Omega$ above $D_{z}$, and $\Omega^{-}$the portion of $\Omega$ below $D_{z}$, with $\partial \Omega^{-}$the portion of $\partial \Omega$ below $D_{z}$. Then, the divergence theorem gives

$$
\begin{aligned}
& \int_{D_{z}} w^{2} d A-\int_{\partial \Omega^{+}} w^{2} v_{3} d s=-2 \int_{\Omega^{+}} w w_{, 3} d x, \\
& \int_{D_{z}} w^{2} d A+\int_{\partial \Omega^{-}} w^{2} v_{3} d s=2 \int_{\Omega^{-}} w w_{, 3} d x .
\end{aligned}
$$

Combining (2.20) and (2.21) and making use of Schwarz's inequality, we obtain

$$
\int_{D_{z}} w^{2} d A \leq \frac{1}{2} \int_{\partial \Omega} w^{2} d s+\left[\int_{\Omega} w^{2} d x \int_{\Omega}|\nabla w|^{2} d x\right]^{\frac{1}{2}} .
$$

On the other hand, from the definition of $p_{0}$ (see (2.16)) and the divergence theorem, we have

$$
p_{0} \int_{\partial \Omega} w^{2} d s \leq \int_{\partial \Omega} x_{i} n_{i} w^{2} d s=3 \int_{\Omega} w^{2} d x+2 \int_{\Omega} x_{i} w w_{, i} d x,
$$

so that we obtain

$$
\int_{\partial \Omega} w^{2} d s \leq \frac{3}{p_{0}} \int_{\Omega} w^{2} d x+\frac{2 d}{p_{0}}\left[\int_{\Omega} w^{2} d x \int_{\Omega}|\nabla w|^{2} d x\right]^{\frac{1}{2}} .
$$

Therefore, replacing (2.24) in (2.22), we get

$$
\int_{D_{z}} w^{2} d A \leq \frac{3}{2 p_{0}} \int_{\Omega} w^{2} d x+\left(1+\frac{d}{p_{0}}\right)\left[\int_{\Omega} w^{2} d x \int_{\Omega}|\nabla w|^{2} d x\right]^{\frac{1}{2}} .
$$

Going back to (2.19) we find, after some manipulations, that

$$
\begin{aligned}
\left(\int_{\Omega} w^{4} d x\right)^{\frac{1}{2}} & \leq \frac{3}{2}\left(\int_{\Omega} w^{2} d x\right)^{\frac{1}{4}}\left\{\frac{3}{2 p_{0}}\left(\int_{\Omega} w^{2} d x\right)^{\frac{1}{2}}+\left(1+\frac{d}{p_{0}}\right)\left(\int_{\Omega}|\nabla w|^{2} d x\right)^{\frac{1}{2}}\right\}^{\frac{3}{2}} \\
& =\frac{3}{2}\left\{\frac{3}{2 p_{0}}\left(\int_{\Omega} w^{2} d x\right)^{\frac{2}{3}}+\left(1+\frac{d}{p_{0}}\right)\left(\int_{\Omega} w^{2} d x\right)^{\frac{1}{6}}\left(\int_{\Omega}|\nabla w|^{2} d x\right)^{\frac{1}{2}}\right\}^{\frac{3}{2}} .
\end{aligned}
$$


Next, with $w:=u^{n}$, we replace (2.26) in (2.5) to obtain

$$
\begin{aligned}
\int_{\Omega} u^{2 n+p-1} d x \leq & \left(\frac{3}{2}\right)^{\frac{2}{3}}|\Omega|^{\frac{2}{3}(1-\mu)} \Phi(t)^{\frac{2}{3} \mu} \\
& \times\left\{\frac{3}{2 p_{0}} \Phi(t)^{\frac{2}{3}}+\left(1+\frac{d}{p_{0}}\right) \Phi(t)^{\frac{1}{6}}\left(\int_{\Omega}\left|\nabla u^{n}\right|^{2} d x\right)^{\frac{1}{2}}\right\} .
\end{aligned}
$$

Moreover, making use of the inequality $a b \leq \frac{a^{2}}{2 \alpha}+\frac{b^{2} \alpha}{2}$, where $\alpha$ is an, as yet, unspecified positive weight to be chosen, we have

$$
\Phi(t)^{\frac{4 \mu+1}{6}}\left(\int_{\Omega}\left|\nabla u^{n}\right|^{2} d x\right)^{\frac{1}{2}} \leq \frac{1}{2 \alpha} \Phi(t)^{\frac{4 \mu+1}{3}}+\frac{\alpha}{2} \int_{\Omega}\left|\nabla u^{n}\right|^{2} d x .
$$

Therefore, replacing (2.28) in (2.27) and, thereafter, (2.27) in (2.4), we get

$$
\begin{aligned}
\Phi^{\prime}(t) \leq & -\frac{2 n(2 n-1)}{n^{2}} b_{1} \int_{\Omega}\left|\nabla u^{n}\right|^{2} d x+2 n a_{1}|\Omega|^{\frac{1}{2 n}} \Phi(t)^{\frac{2 n-1}{2 n}}+n a_{2} \\
& \times\left(\frac{3^{5}}{2^{2}}\right)^{\frac{1}{3}} \frac{1}{p_{0}}|\Omega|^{\frac{2}{3}(1-\mu)} \Phi(t)^{\frac{2}{3}(1+\mu)}+n a_{2}\left(\frac{3}{2}\right)^{\frac{2}{3}} \frac{1}{\alpha}\left(1+\frac{d}{p_{0}}\right)|\Omega|^{\frac{2}{3}(1-\mu)} \Phi(t)^{\frac{4 \mu+1}{3}} \\
& +n a_{2} \alpha\left(\frac{3}{2}\right)^{\frac{2}{3}}\left(1+\frac{d}{p_{0}}\right)|\Omega|^{\frac{2}{3}(1-\mu)} \int_{\Omega}\left|\nabla u^{n}\right|^{2} d x
\end{aligned}
$$

Choosing now the parameter $\alpha$ in (2.29) such that

$$
-\frac{2 n(2 n-1)}{n^{2}} b_{1}+n a_{2} \alpha\left(\frac{3}{2}\right)^{\frac{2}{3}}\left(1+\frac{d}{p_{0}}\right)|\Omega|^{\frac{2}{3}(1-\mu)}=0,
$$

we obtain the following differential inequality for $\Phi(t)$ :

$$
\Phi^{\prime}(t) \leq K_{1} \Phi(t)^{\frac{2 n-1}{2 n}}+K_{2} \Phi(t)^{\frac{2}{3}(1+\mu)}+K_{3} \Phi(t)^{\frac{4 \mu+1}{3}},
$$

where

$$
\begin{aligned}
& K_{1}:=2 n a_{1}|\Omega|^{\frac{1}{2 n}}, K_{2}:=n a_{2}\left(\frac{3^{5}}{2^{2}}\right)^{\frac{1}{3}} \frac{1}{p_{0}}|\Omega|^{\frac{2}{3}(1-\mu)}, \\
& K_{3}:=n a_{2}\left(\frac{3}{2}\right)^{\frac{2}{3}} \frac{1}{\alpha}\left(1+\frac{d}{p_{0}}\right)|\Omega|^{\frac{2}{3}(1-\mu)} .
\end{aligned}
$$

Next, an integration of the differential equation (2.31) from 0 to $t$ gives

$$
\int_{\Phi(0)}^{\Phi(t)} \frac{d \eta}{K_{1} \eta^{\frac{2 n-1}{2 n}}+K_{2} \eta^{\frac{2}{3}(1+\mu)}+K_{3} \eta^{\frac{4 \mu+1}{3}}} \leq t .
$$

Therefore, if $u(\mathbf{x}, t)$ blows up in the measure $\Phi$ as $t \longrightarrow t^{*}$, we obtain the lower bound

$$
t^{*} \geq \int_{\Phi(0)}^{\infty} \frac{d \eta}{K_{1} \eta^{\frac{2 n-1}{2 n}}+K_{2} \eta^{\frac{2}{3}(1+\mu)}+K_{3} \eta^{\frac{4 \mu+1}{3}}},
$$


where $\mu$ was given in (2.6). Clearly, since $2(\mu+1) / 3>1$ and $(4 \mu+1) / 3>1$, the integral in (2.34) is bounded.

We summarise this result in the following theorem:

THEOREM. If $n>3(p-1) / 2$ and $u(\mathbf{x}, t)$ is a non-negative classical solution of the problem (1.1)-(1.3), which becomes unbounded at time $t=t^{*}$ in the measure $\Phi(t)$ given by (2.1), then $t^{*}$ is bounded below by (2.34), where $K_{1}, K_{2}$ and $K_{3}$ are given in (2.32).

ACKNOWLEDGEMENTS. The author would like to thank professor Lawrence E. Payne for fruitful discussions and hospitality during his visit at Cornell University (USA), where this research was initiated. This work was supported by a CNCSIS PN-II-RU-RP research grant (No. 4/3.11.2008).

\section{REFERENCES}

1. J. M. Ball, Remarks on blow-up and non-existence theorems for nonlinear evolution equations, Q. J. Math. 28 (1977), 473-486.

2. V. A. Galaktionov and J. L. Vazquez, The problem of blow-up in nonlinear parabolic equations, Discrete Cont. Dyn. Syst. 8(2) (2002), 399-433.

3. H. Kielhöfer, Existenz und Regularität von Lösungen semilinearer parabolischer Anfangs-Randwertprobleme, Math. Z. 142 (1975), 131-160.

4. H. A. Levine, Nonexistence of global weak solutions to some properly and improperly posed problems of mathematical physics. The method of unbounded Fourier coefficients, Math. Ann. 214 (1975), 205-220.

5. L. E. Payne, G. A. Philippin and P. W. Schaefer, Blow-up phenomena for some nonlinear parabolic problems, Nonlinear Anal. 69 (2008), 3495-3502.

6. L. E. Payne and P. W. Schaefer, Lower bounds for blow-up time in parabolic problems under Neumann conditions, Appl. Anal. 85 (2006), 1301-1311.

7. L. E. Payne and P. W. Schaefer, Lower bounds for blow-up time in parabolic problems under Dirichlet conditions, J. Math. Anal. Appl. 328 (2007), 1196-1205.

8. L. E. Payne and P. W. Schaefer, Bounds for the blow-up time for the heat equation under nonlinear boundary conditions, Proc. Roy. Soc. Edinburgh Sect. A. 139(6) (2009), 1289-1296.

9. L. E. Payne and J. C. Song, Lower bounds for blow-up time in a nonlinear parabolic problem, J. Math. Anal. Appl. 354 (2009), 294-396.

10. M. H. Protter and H. F. Weinberger, Maximum principles in differential equations (Springer-Verlag, Berlin, 1975).

11. R. Quittner and P. Souplet, Superlinear parabolic problems. Blow-up, global existence and steady states (Birkhauser, Basel, 2007).

12. B. Straughan, Explosive instabilities in mechanics (Springer-Verlag, Berlin, 1998). 